\title{
Anti-rheumatic drug iguratimod protects against cancer-induced bone pain and bone destruction in a rat model
}

\author{
YUE SUN $^{1}$, YING-XING WU ${ }^{2}$, PENG ZHANG ${ }^{1}$, GUANG PENG $^{3}$ and SHI-YING YU ${ }^{1}$ \\ ${ }^{1}$ Cancer Center; ${ }^{2}$ Department of Orthopedics, Tongji Hospital, Tongji Medical College, Huazhong \\ University of Science and Technology, Wuhan, Hubei 430030, P.R. China; ${ }^{3}$ Department of Clinical \\ Cancer Prevention, MD Anderson Cancer Center, University of Texas, Houston, TX 77030, USA
}

Received August 21, 2015; Accepted January 26, 2017

DOI: $10.3892 / 01.2017 .6045$

\begin{abstract}
The bone is one of the most common sites of metastasis in patients with cancer. Current treatments for bone metastases include bisphosphonates, denosumab, non-steroidal anti-inflammatory drugs and analgesics, but each of them has certain limitations. Cytokines and mediators released from various cells in the bone microenvironment may drive a vicious cycle of osteolytic bone metastases. Iguratimod (T-614), a novel disease-modifying anti-rheumatic drug, has demonstrated therapeutic effects by suppressing the production of inflammatory cytokines in rats and patients with rheumatoid arthritis. Therefore, the current study evaluated the hypothesis that iguratimod may protect against cancer-induced bone pain and bone metastasis in a rat model. For this purpose, rats inoculated with Walker 256 cells were treated with iguratimod from days 11-17 post-surgery. Mechanical paw withdrawal thresholds and expression levels of phosphorylated extracellular signal-related kinase (pERK) and c-Fos in the spinal cord were investigated to detect changes in bone pain. Bone destruction levels were detected using X-rays, hematoxylin and eosin and tartrate-resistant acid phosphatase staining. The results revealed that mechanical paw withdrawal thresholds and the expression levels of pERK and c-Fos declined in a dose-dependent manner in rats treated with iguratimod, and bone destruction severity was also reduced. These findings may provide important new insights into the treatment of bone metastasis symptoms.
\end{abstract}

\section{Introduction}

Bone is one of the most common sites of metastases in patients with cancer (1). It is reported that $\sim 75 \%$ of women

Correspondence to: Dr Shi-Ying Yu, Cancer Center, Tongji Hospital, Tongji Medical College, Huazhong University of Science and Technology, 1095 Jiefang Road, Wuhan, Hubei 430030, P.R. China

E-mail: syyu@tjh.tjmu.edu.cn

Key words: bone metastasis, bone cancer pain, bone destruction, rat model, iguratimod, protection with advanced breast cancer develop bone metastasis $(2,3)$. Bone metastasis frequently results in skeletal-related events, including severe bone pain, pathological fraction, spinal cord compression and the requirement for surgery or radiotherapy, which may be associated with decreased quality of life and poor prognosis $(1,2,4)$.

Current treatments for bone metastases include bisphosphonates, denosumab, non-steroidal anti-inflammatory drugs (NSAIDs) and analgesics, but each of them has certain limitations (5). Bisphosphonates, which are recommended for bone metastasis treatment, are associated with the occasional development of osteonecrosis of the jaw (6). Denosumab is superior to zoledronic acid in reducing skeletal-related events in patients with bone metastasis, but hypocalcemia occurs more frequently in patients receiving denosumab (7). NSAIDs are frequently considered to be more efficacious in reducing bone cancer pain compared with other pain states, but they are associated with gastrointestinal injury and myocardial infarction $(5,8)$. In this regard, alternative drugs that are able to assist the treatment for bone metastasis are required.

Osteolytic bone metastases are considered to derive from a 'vicious cycle' of progressive interactions between tumor cells and the bone microenvironment (9). In this microenvironment, large quantities of cytokines and mediators, which are released from tumor cells, osteocytes and degraded bone matrix promote the process of bone resorption $(1,10,11)$.

Iguratimod (T-614), a novel disease-modifying anti-rheumatic drug, has exhibited anti-rheumatic effects through suppression of the production of inflammatory cytokines, including tumor necrosis factor- $\alpha$ (TNF- $\alpha$ ), interleukin (IL)-1 $\beta$, IL-6, IL-8 and IL-17, and immunoglobulins, as well as inhibiting the activation of nuclear factor-kappa $\mathrm{B}(\mathrm{NF}-\kappa \mathrm{B})(12,13)$. As cytokines are involved in the process of bone metastasis, the present study evaluated the hypothesis that iguratimod may protect against cancer-induced bone pain and bone destruction, potentially via anti-inflammatory effects in a rat model. The findings may have the potential to rapidly translate into treatment strategies for patients with bone metastasis.

\section{Materials and methods}

Animals. Female Wistar rats (180-200 g, Tongji Hospital, Huazhong University of Science and Technology, Wuhan, 
China) were maintained at a temperature of $22 \pm 1^{\circ} \mathrm{C}$ under a 12-h/12-h light-dark cycle regime with free access to food and water. All experimental protocols were approved by the Medical Ethics Committee of Huazhong University of Science and Technology and were performed according to the ethical guidelines of the National Institutes of Health Guide for Care and Use of Laboratory Animals.

Preparation of carcinoma cells. Walker 256 rat mammary gland carcinoma cells were provided by the Department of Anesthesiology at Tongji Hospital (Huazhong University of Science and Technology, Wuhan, China) and cultured at $37^{\circ} \mathrm{C}$, in an atmosphere containing $5 \% \mathrm{CO}_{2}$ in RPMI 1640 medium (Gibco; Thermo Fisher Scientific, Inc., Waltham, MA, USA) containing $10 \%$ fetal bovine serum (Gibco; Thermo Fisher Scientific, Inc.), $100 \mathrm{U} / \mathrm{ml}$ penicillin and $100 \mu \mathrm{g} / \mathrm{ml}$ streptomycin (Wuhan Boster Biological Technology, Ltd., Wuhan, China). The cells were rinsed twice with calcium-and magnesium-free PBS solution and collected by centrifuging the medium for $5 \mathrm{~min}$ at $200 \mathrm{x} \mathrm{g}$. The pellet was subsequently re-suspended in PBS solution and the concentration was adjusted to $8 \times 10^{6}$ cells $/ \mathrm{ml}$ using a hemocytometer. The cell suspension was maintained on ice until inoculation.

Bone cancer pain model. The procedure was performed as previously described $(14,15)$. Briefly, the rats were completely anesthetized with $10 \%$ chloral hydrate $(3 \mathrm{ml} / \mathrm{kg}$, intraperitoneal) and placed in the supine position. The left leg was shaved and the skin was disinfected with $7 \%$ iodine. The top half of the tibia was exposed with minimum damage. A 23-gauge needle was inserted into the intramedullary canal of tibia, $7 \mathrm{~mm}$ distal to the epiphyseal growth plate below the knee joint. Then the needle was removed and replaced with a $25 \mu \mathrm{l}$ Hamilton syringe containing the cells $\left(10 \mu 1,8 \times 10^{4}\right.$ cells $)$ or vehicle (PBS solution). Following slow injection and $3 \mathrm{~min}$ retention, the Hamilton syringe was removed and the drilled hole was immediately sealed with bone wax. The site was thoroughly washed with sterile deionized water and infiltrated with gentamicin. The muscle and skin were finally sutured and disinfected. The rats were returned to their room cages following regaining consciousness.

Drug treatments. Iguratimod was provided by Simcere Pharmaceutical Group (Nanjing, China). The drug was suspended in $0.5 \%$ methylcellulose solution. Iguratimod (daily dose 5 or $20 \mathrm{mg} / \mathrm{kg})$ or vehicle $(0.5 \%$ methylcellulose solution) was administered orally once daily from day 11 after the tumor cell inoculation (day 0) for 7 days (16).

Mechanical allodynia test. Each rat was tested for mechanical allodynia prior to the injection of cancer cells or sham, and again on days 4, 8, 12 and 16 post-surgery. Animals were placed in individual plastic boxes with a metal mesh floor and allowed to habituate for $30 \mathrm{~min}$ prior to tests. Mechanical paw withdrawal threshold was measured by an ascending series of von Frey filaments (0.6, 1.0, 1.4, 2.0, 4.0, 6.0, 8.0, 10.0 and $15.0 \mathrm{~g}$; Stoelting, Wood Dale, IL, USA) as previously reported $(14,17,18)$. The filaments were applied perpendicular to mid-plantar surface of the left hind paw.
Each hair was held for $\sim 1-2 \mathrm{sec}$ with a $10 \mathrm{sec}$ interval and was applied 5 times per filament. The test was initiated with the application of the $2.0 \mathrm{~g}$ hair and the positive response was defined as a quick withdrawal or paw flinching. Whenever a positive response was performed, the next lowest hair was applied and whenever a negative response occurred, a higher hair was applied. The paw withdrawal frequency (PWF) to each monofilament was calculated from five applications. Paw withdrawal threshold (PWT) was considered the force at which $\mathrm{PWF} \geq 60 \%$; $15 \mathrm{~g}$ was recorded as the PWT if $\mathrm{PWF}<60 \%$ to all filaments (18).

Western blot analysis. Rats were sacrificed on day 17, 4-6 h after drug treatments. The whole spinal cord at L2-L5 segments was quickly removed and the total protein was extracted using Trizol $^{\circledR}$ reagent (Invitrogen; Thermo Fisher Scientific, Inc.). Protein concentrations were measured using a bicinchoninic acid assay kit (Beyotime Institue of Biotechnology, Guangzhou, China), and protein samples were heated for 5 min at $100^{\circ} \mathrm{C}$ with SDS-PAGE sample buffer (Wuhan Boster Biological Technology, Ltd.). Subsequently, the equivalent amounts of protein samples $(30 \mu \mathrm{g})$ were separated by $10 \%$ SDS-PAGE electrophoresis and subsequently transferred onto polyvinylidene difluoride membranes. The membranes were blocked in $5 \%$ bovine serum albumin containing $0.1 \%$ Tween-20 at room temperature for $1 \mathrm{~h}$ and incubated overnight at $4^{\circ} \mathrm{C}$ with primary antibodies against phosphorylated extracellular signal-related kinase (pERK) 1/2 (dilution, 1:1,000; \#4370; Cell Signaling Technology, Inc., Danvers, MA, USA), extracellular signal-related kinase (ERK) 1/2 (dilution, 1:1,000; \#9120; Cell Signaling Technology, Inc.), c-Fos (dilution, 1:1,000; \#sc-52; Santa Cruz Biotechnology, Inc., Dallas, TX, USA) or GAPDH (dilution, 1:2,000; \#PB0141, Wuhan Boster Biological Technology, Ltd.). Subsequently, the membranes were washed in Tris-buffered saline containing $0.1 \%$ Tween-20 and incubated with the secondary antibody conjugated with horseradish peroxidase (dilution, 1:2,000; \#BA1054, Wuhan Boster Biological Technology, Ltd) for $1 \mathrm{~h}$ at room temperature. Membranes were visualized with Pierce Super Signal West Pico Chemiluminescent Substrate (Pierce; Thermo Fisher Scientific, Inc.). Images were captured with the ChemiDoc $^{\mathrm{TM}} \mathrm{XRS}+$ imaging system (Bio-Rad Laboratories, Inc., Hercules, CA, USA). The protein expression was normalized to GAPDH or total proteins presented in the corresponding lane on the membrane using Image Lab software, version 5.1 (Bio-Rad Laboratories, Inc.).

$X$-ray test. Left hind limbs were collected from cadavers and assessed radiologically on day 17 prior to decalcification and histological staining. Hind limbs were exposed to an X-ray source for $80.0 \mathrm{msec}$ at $55 \mathrm{kV}$ (DR-F, GE Hualun Medical Systems, Beijing, China). Radiological scores to evaluate the bone destruction of each tibia were determined based on blind analysis of radiographs, using a previously published system $(19,20)$. All scores are associated with the tibia (bone): 0 , normal bone structure without any sign of deterioration; 1 , small radiolucent lesions in the proximal epiphysis $(<3)$, close to the site of the injection; 2 , increased number of radiolucent lesions (>3) loss of medullary bone; 3 , loss of medullary bone, plus erosion of the cortical bone; 4 , full thickness unicortical 
bone loss; 5 , full thickness bicortical bone loss and displaced fractures $(19,20)$.

Histological staining. For histological staining, rat tibiae were gently separated and fixed in $4 \%$ paraformaldehyde for 2 days. Following decalcification in 10\% EDTA for 2 weeks, the tibiae were embedded and stained with Harris' hematoxylin and eosin (H\&E) to determine cancer cell infiltration. Subsequently, decalcified slices were stained using tartrate-resistant acid phosphatase (TRAP) according to the manufacturer's protocol (Nanjing Jiancheng Biological Engineering Research Institute, Nanjing, China). Osteoclasts were defined as TRAP-positive cells containing $\geq 3$ nuclei, as counted under a light microscope (TE2000; Nikon Corporation, Tokyo, Japan). Five randomly selected fields under x400 magnification were examined to count TRAP (+) cells in each group. Analysis was performed in a blinded fashion.

Quantitative analysis of plasma IL-6 level. Rats' blood was obtained using left ventricular puncture with syringes containing heparin on day 17 post-surgery. Plasma was separated following centrifugation. The quantitation of IL- 6 in plasma was performed using the rat IL-6 ELISA kit according to the manufacturer's protocol (Dakewe Biotech Co., Ltd., Beijing, China).

Statistical analysis. Statistical analysis was performed using SPSS software, version 19.0 (IBM SPSS, Armonk, NY, USA). All data are expressed as the mean \pm standard error of the mean. Statistical analyses between two samples were performed using the Student's $t$-test. Statistical comparison of more than two groups was performed using one-way analysis of variance followed by a Tukey test. Data from the behavior test and X-ray scores were analyzed across treatment groups using a Kruskal-Wallis nonparametric analysis of variance test. $\mathrm{P}<0.05$ was considered to indicate a statistically significant difference.

\section{Results}

Analgesic effects of iguratimod. The analgesic effect of iguratimod was investigated using animal models. Fig. 1 reveals that iguratimod significantly improved the pain withdrawal threshold of the left hind paw in dose-dependent manner.

The mechanical PWT of each rat was tested prior to injections and every 4 days following the surgery. The PWT of tumor-free rats remained at a high level throughout the test, but the PWT of tumor-bearing rats decreased from day 4 post-surgery and reached a low level at day 8. Iguratimod and vehicle were administered to groups from days 11-17. From day 12 , the PWT of rats with iguratimod exhibited an upward trend and it increased more significantly in the high dose group (20 mg/kg), whereas the PWT of tumor-bearing rats treated with vehicle maintained a downward trend. On day 16 , the PWT of rats in the high dose group was significantly higher compared with the vehicle group $(4.60 \pm 0.98$ vs. $0.80 \pm 0.33$ g; $\mathrm{P}<0.05)$. Low dose iguratimod $(5 \mathrm{mg} / \mathrm{kg})$ also exhibited a degree of analgesic effect, but the difference in PWT between the vehicle group and the low dose group at day 16 was not statistically significant.

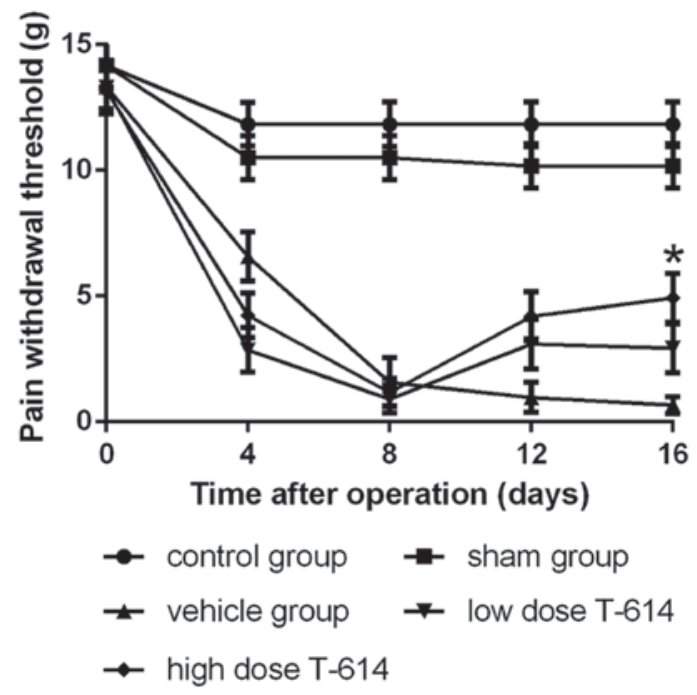

Figure 1. Iguratimod attenuated the mechanical allodynia induced by cancer cell inoculation. Iguratimod was administrated from day 11-17 and behavioral tests were conducted at days $0,4,8,12$ and 16. The PWT of rats in vehicle group continued to decrease following inoculation. The PWT of rats treated with iguratimod decreased prior to drug treatment and presented an upward trend in a dose-dependent manner following treatment. ${ }^{*} \mathrm{P}<0.05$, compared with the vehicle group; $n=6$ rats/group.

Effects of iguratimod on pERK and c-Fos expression in spinal cord. To determine whether bone cancer pain was mitigated by iguratimod, proteins that are associated with bone cancer pain in the spinal cord were investigated. As pERK and c-Fos are markers for neuronal activation and central sensitization, the protein levels of pERK1/2 and c-Fos in the spinal cord were evaluated using western blot analysis (21-24). As presented in Fig. 2, the protein levels of spinal pERK1/2 were increased in tumor-bearing rats at day 17, whereas the levels were lower in cancer-free rats. Furthermore, the pERK1/2 levels declined in a dose-dependent manner when iguratimod was administered and the difference between the vehicle group and high dose group was statistically significant. No significant effect was observed on the total ERK1/2 levels. The same trend in c-Fos levels in the spinal cords was detected and the difference between the vehicle group and high dose group was also statistically significant (Fig. 2). Alterations in pERK1/2 and c-Fos were concordant with the trends in mechanical PWT.

Effect of iguratimod on bone resorption. A total of 17 days after injection of Walker 256 rat mammary cancer cells into the intramedullary space of the rat tibia, tumor growth and bone resorption were observed in tumor-bearing tibiae. Serial sections stained with H\&E demonstrated that cancer cells grew invasively in the bone marrow cavity and the trabecular bone was damaged significantly in the vehicle group at day 17 post-surgery. However, trabecular bone destruction was lighter and some normal trabecular bone was observed in rats treated with iguratimod. Bone resorption was not observed in the sham group (Fig. 3).

$\mathrm{X}$-ray tests were conducted for each left hind paw at day 17 after inoculation and the scores of bone destruction were calculated. As presented in Fig. 4, varying degrees 


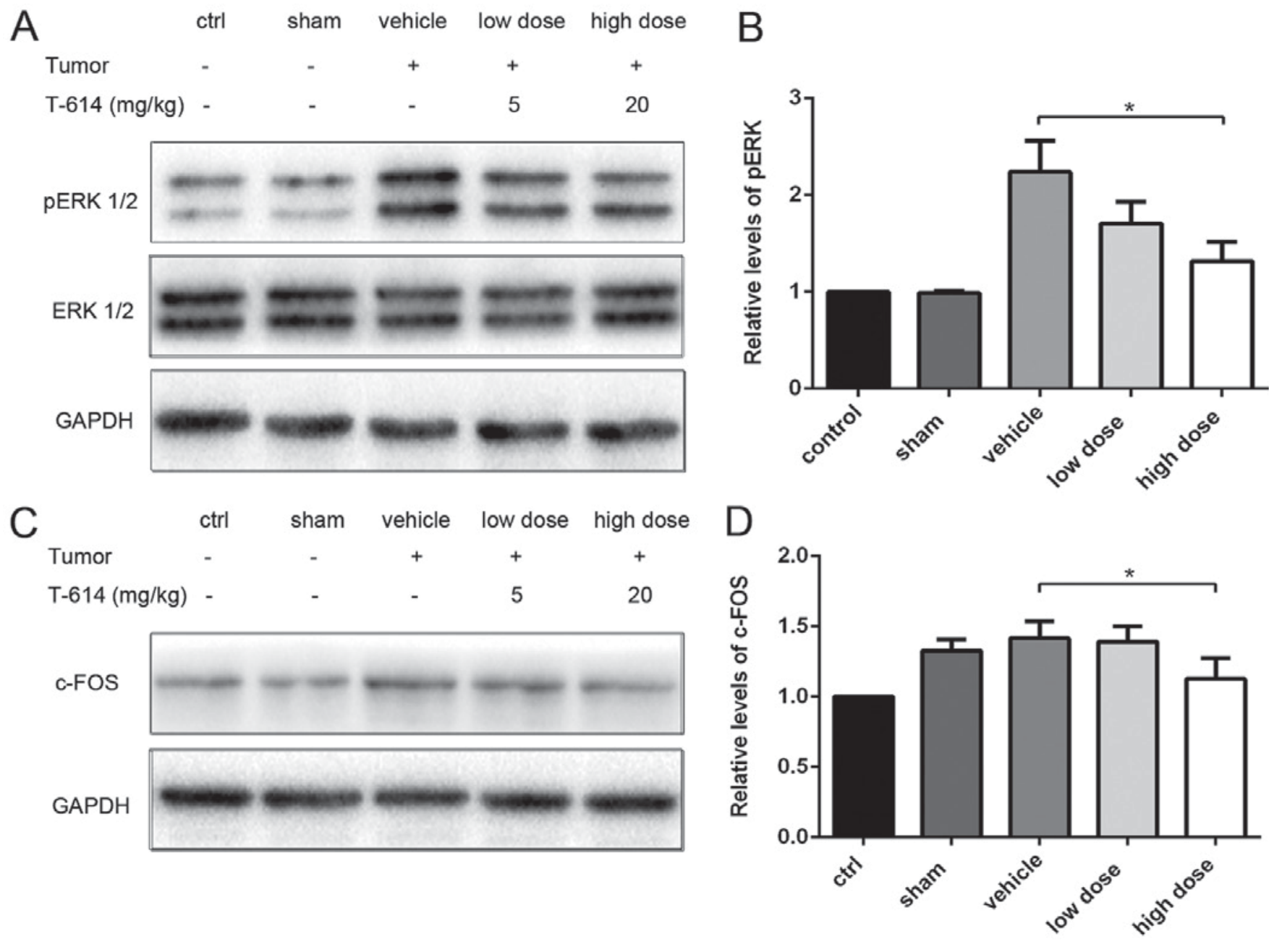

Figure 2. Iguratimod reduced the elevation of pERK1/2 and c-Fos in the spinal cord induced by cancer cell inoculation. (A and B) The representative bands for the expression of pERK1/2 in the spinal cord following inoculation and the quantitative data for the expression of pERK1/2 (detected by western blot analysis). The fold change for the density of pERK1/2 was normalized to ERK1/2 for each sample respectively. (C and D) The representative bands for the expression of c-Fos in the spinal cord following inoculation and the quantitative data for the expression of c-Fos (detected by western blot analysis). The fold change for the density of c-Fos was normalized to GAPDH for each sample respectively. ${ }^{*} \mathrm{P}<0.05$, compared with vehicle group; $\mathrm{n}=3$ rats/group. pERK, phosphorylated extracellular signal-related kinase; ERK, extracellular signal-related kinase; T-614, iguratimod; ctrl, control.

of bone destruction were detected in the proximal tibiae of tumor-bearing rats, whereas these phenomena were not observed in the sham group. Rats from the vehicle group presented multiple radiation translucent areas due to medullary bone loss, as well as bilateral cortical defects. However, fewer radiolucent lesions were detected in rats treated with iguratimod, and bicortical bone loss was less common compared with the vehicle group; however, unicortical defects may still exist. According to the scores calculated from each group, the scores of the high dose group were significantly lower than those of the vehicle group $(1.6 \pm 0.50$ vs. $3.33 \pm 0.58$, respectively).

As bone destruction is associated with the increased activity of osteoclasts, the activation of osteoclasts was detected using TRAP staining. As observed under the microscope, osteoclasts were claret-colored multinucleated cells primarily distributed along the edges of trabeculae in the tibia metaphysis. As presented in Fig. 5, osteoclasts were rarely identified in the tibiae of the sham group. However, the number of TRAP (+) multinucleated cells was significantly increased in the vehicle group. In rats treated with iguratimod, the activity of osteoclasts appeared weaker than that of the vehicle group, resulting in fewer TRAP (+) multinucleated cells being identified in stained sections. Five fields were randomly selected under x400 magnification, and TRAP (+) cells were counted for each group. The number of activated osteoclasts in the high dose group was significantly lower than that in the vehicle group (4.47 \pm 2.61 vs. $12.67 \pm 3.95$, respectively).

Effect of iguratimod on plasma levels of IL-6. The plasma IL-6 levels of rats in each group were detected following drug treatment using ELISA analysis. As presented in Fig. 6, plasma IL-6 levels of rats in the vehicle group were highly increased compared with those in the sham group $(394.76 \pm 36.67$ vs. $128.93 \pm 30.35 \mathrm{pg} / \mathrm{ml}$, respectively). The IL-6 levels in rats treated with iguratimod were decreased in a dose-dependent manner compared with those in the vehicle group $(249.10 \pm 31.73$ vs. $394.76 \pm 36.67 \mathrm{pg} / \mathrm{ml} ; 198.09 \pm 33.73$ vs. $394.76 \pm 36.67 \mathrm{pg} / \mathrm{ml}$, respectively) and were correlated positively with the changes in mechanical allodynia and bone destruction in individual animals. 

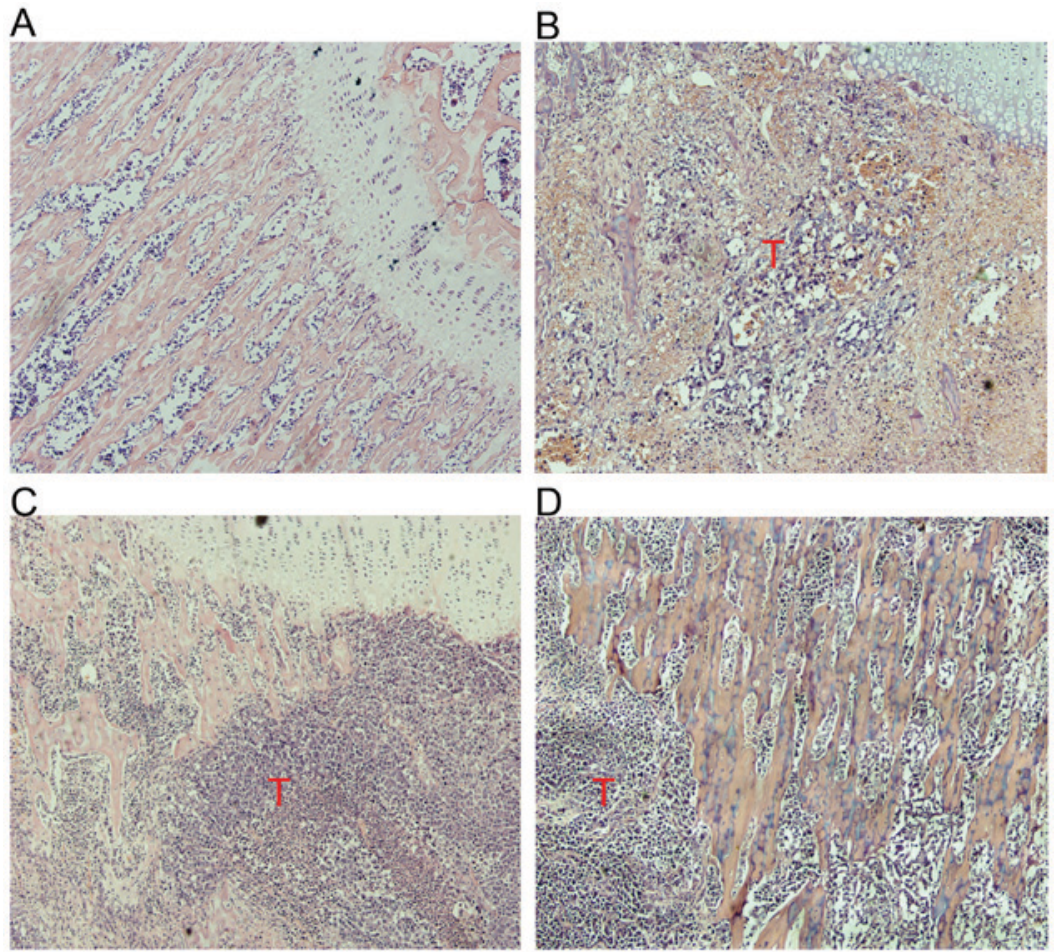

Figure 3. Hematoxylin and eosin staining in the rat tibiae at day 17 post-surgery. (A) The healthy bone structures of the tibiae in the sham group. (B) Breast cancer cells grew invasively in the bone marrow cavity and the trabecular bone was damaged significantly in the vehicle group. (C and D) Trabecular bone destruction was lower and more normal trabecular bone was able to be observed in the low and high dose iguratimod-treated group. Original magnification, $\mathrm{x} 100$; T, Tumor.

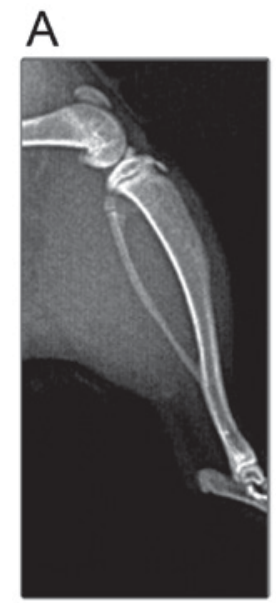

sham

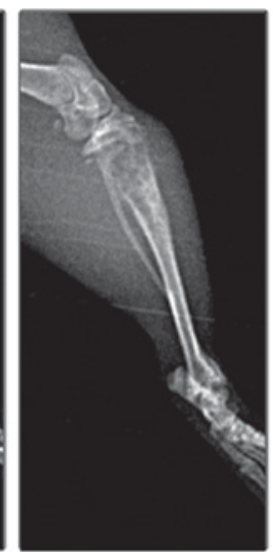

vehicle

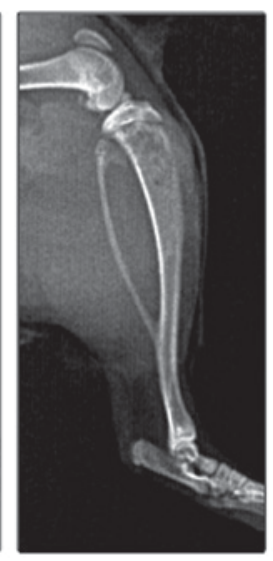

low dose

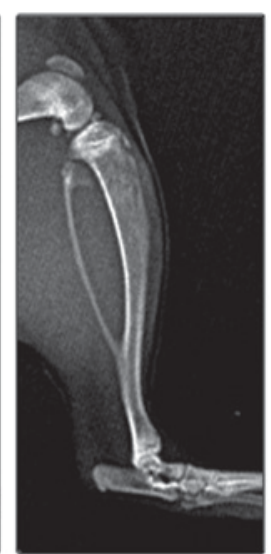

high dose

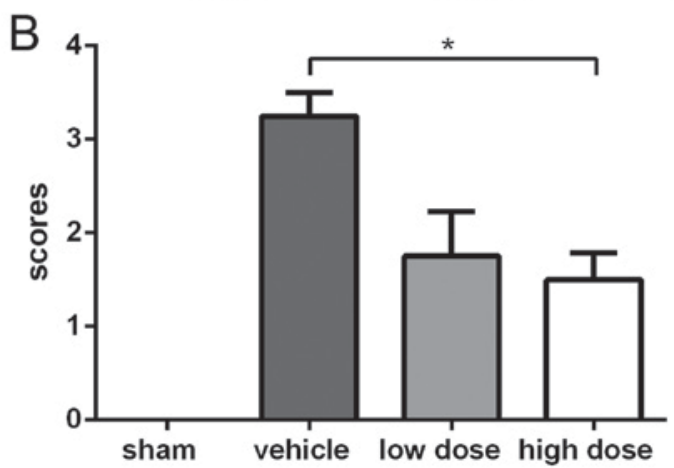

Figure 4. Effects of iguratimod on bone resorption, as evaluated by X-rays. (A) X-ray images of rat tibiae. Tibiae in the sham group had healthy bone structures, whereas multiple radiation translucent areas and bicortical defects were detected in the vehicle group. When iguratimod was administered, bone destruction was reduced. Radiolucent lesions were reduced and bicortical defects were rare. (B) X-ray scores were evaluated according to a previously published system. The scores of the high dose group were significantly lower than those of the vehicle group. * $\mathrm{P}<0.05$, compared with the vehicle group; $\mathrm{n}=3$ rats/group. 
A

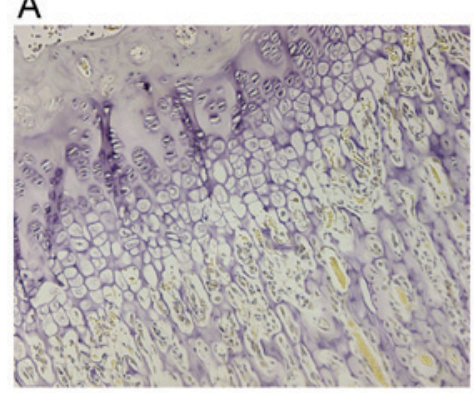

C

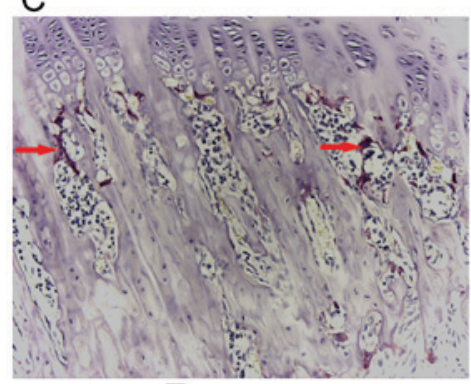

B

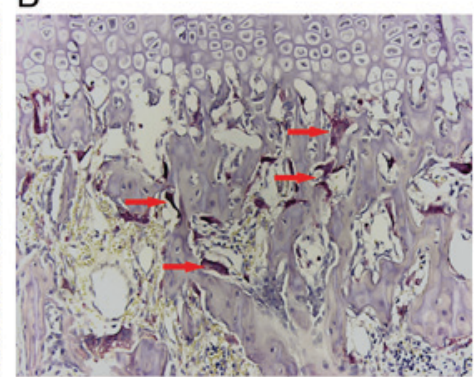

D

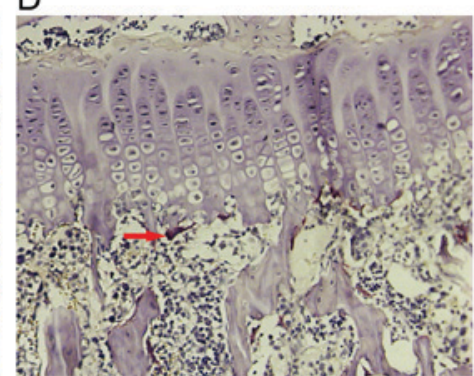

$\mathrm{E}$

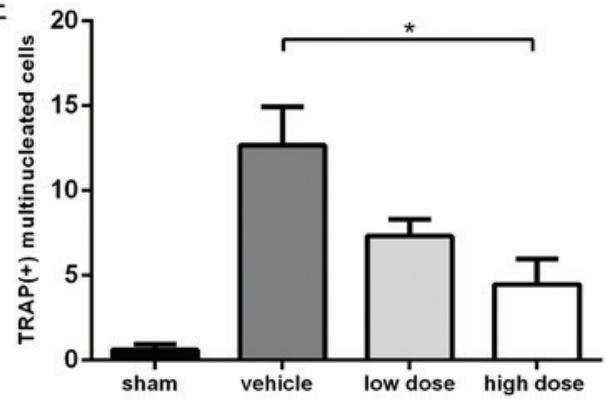

Figure 5. Effects of iguratimod on osteoclastogenesis. (A) Osteoclasts were rarely detected in the tibiae of the sham group. (B) The number of TRAP (+) multinucleated cells was significantly increased in the vehicle group. (C) Fewer TRAP (+) cells were found in rat tibiae in the low dose iguratimod group. (D) The number of osteoclasts was reduced when rats were treated with high dose igutarimod. (E) Five fields were randomly selected under $\mathrm{x} 400$ magnification and TRAP (+) cells were counted for each group. The number of activated osteoclasts in the high dose group was significantly reduced compared with the vehicle group. ${ }^{*} \mathrm{P}<0.05$, compared with the vehicle group; $\mathrm{n}=3$ rats/group. Original magnification, $\mathrm{x} 200$. Osteoclasts are marked with red arrows. TRAP, tartrate-resistant acid phosphatase.

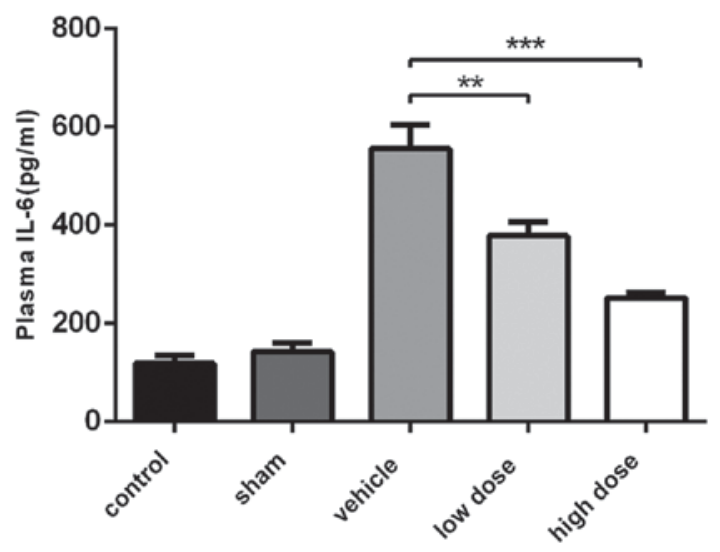

Figure 6. Effect of iguratimod on plasma levels of IL-6. IL-6 levels in the vehicle-treated rats were highly increased in the plasma compared with the control and sham. When treated with iguratimod, the IL-6 levels were significantly decreased in a dose-dependent manner. ${ }^{* *} \mathrm{P}<0.01$ and ${ }^{* * * *} \mathrm{P}<0.001$, compared with vehicle group, $\mathrm{n}=3$ rats/group. IL-6, interleukin-6.

\section{Discussion}

The Walker 256 rat mammary carcinoma cell-induced bone cancer pain model has been extensively utilized to elucidate the underlying mechanisms for cancer-induced bone pain. In the current study, the anti-nociceptive effect of iguratimod was investigated in this rat model.

The mechanical PWT was used to detect the analgesic effect of iguratimod. In the vehicle-treated animals, the PWT declined throughout the study, but when iguratimod was administered, the PWT exhibited an upward trend. The changes in the expression levels of biomarkers in the spinal cord associated with bone cancer pain were coincident with those of mechanical PWT. The current study also revealed that iguratimod reduced the bone destruction resulting from cancer cell invasion, using X-ray analysis and TRAP staining. As the plasma IL-6 levels of rats declined in the iguratimod-treated groups, the present study hypothesizes that the efficacy of iguratimod may be associated with its anti-inflammatory effects.

Typically, when bone metastasis occurs, crosstalk between the tumor cells and the bone microenvironment drives a vicious cycle of tumor growth and bone destruction (25-27). In this microenvironment, tumor cells and their associated stromal cells, as well as osteocytes, release large quantities of factors including TNF- $\alpha$, IL- 6 , bradykinin, endothelins, cannabinoids, granulocyte-macrophage colony-stimulating 
factor, nerve growth factor, and parathyroid hormone-related protein (10,28-30). Although pain signals are processed in the nervous system, it is considered that inflammatory mediators and cytokines released from cancer cells, immunocytes, osteoclasts or injured tissues in the local microenvironment are able to stimulate the nociceptor terminals of peripheral afferent sensory neurons $(8,30,31)$. The electrochemical signals converted by local nociceptors are subsequently transmitted to the spinal cord and the central nervous system, and pain sensitivity is enhanced (31). Cytokines in the microenvironment, including like IL-6, IL-1 $\beta$ and TNF- $\alpha$ have an important role in driving bone cancer pain (32). They may directly interact with ion channels and receptors on primary afferent nerves and activate second messengers (protein kinase $\mathrm{C}$, protein kinase A, ERK, c-Jun N-terminal kinases, p38 and mitogen-activated protein kinases) in neurons (33). The phosphorylation states of the receptors and ion channels are subsequently altered and the excitement threshold is reduced (33). When these cytokines affect the primary afferent nerve chronically, transcription factors like cAMP response element binding protein, signal transducer and activator of transcription (STAT) and activating transcription factor-3 may be activated by second messengers and the expression levels of neurotransmitters, peptides and ion channel proteins may be altered (33). This leads to sensitization of the peripheral and central nervous system and results in continued and aggravated pain (33). At present, bone cancer pain is considered to be a mixed-mechanism pain state involving inflammatory, neuropathic, ischemic and cancer-specific mechanisms (34). Anti-inflammatory drugs such as NSAIDs are commonly used as adjuvant drugs to stronger analgesics, so that patients may achieve improved analgesic effects (34). Iguratimod is a novel disease-modifying anti-rheumatic drug. Numerous studies have revealed that, when treated with iguratimod, the levels of inflammatory cytokines (TNF- $\alpha$, IL-1 $\beta$, IL-6, IL-8 and IL-17) are declined in arthritis rats, and their arthritis symptoms are also relieved $(12,16,35)$. The current study therefore hypothesized that its anti-inflammatory effects may also enable it to alleviate bone cancer pain. According to the data in the present study, iguratimod significantly reduced the mechanical pain of tumor-bearing rats in a dose-dependent manner, and the plasma IL-6 levels were also declined in rats treated with iguratimod. This is consistent with the theoretical hypothesis that iguratimod alleviates bone cancer pain by affecting the vicious circle via exerting anti-inflammatory effects. The present findings identified a potential additional beneficial effect of iguratimod in treatment of bone cancer pain.

Iguratimod also demonstrated the effect of protecting against bone destruction in the current study. It is considered that increased activity of osteoclasts induced by tumor cells is the main underlying mechanism responsible for bone destruction (1). According to the data in the present study, osteoclasts were markedly activated in tumor-bearing rats while typical medullary bone loss and cortical defects were also detected in them. Cytokines are reported to be a contributor to the activation of osteoclast precursors (30). For example, IL-6, which is primarily produced by stromal cells in the bone microenvironment, is a strong stimulator of osteoclast formation (36), and enhances bone resorption in numerous ways. Firstly, it induces the production of receptor activator of nuclear factor $\kappa \mathrm{B}$ ligand (RANKL) by bone marrow mesenchymal cells and osteoblasts via the IL-6/STAT3 signaling pathway. Osteoclast differentiation and maturation are therefore increased, resulting in the binding of RANKL to its receptor RANK. Secondly, IL-6 increases the expression levels of several proteins that aggravate bone degradation, such as parathyroid hormone-related protein, IL-8, RANKL and cyclooxygenase-2 (COX-2) in tumor cells. Thirdly, IL- 6 imbalances bone homeostasis towards excessive degradation by inhibiting Wnt-mediated osteogenesis and downregulating the synthesis of genes including type II collagen and aggrecan $(37,38)$. Other cytokines, such as TNF- $\alpha$, IL- $1 \beta$ and IL- 8 also serve an important role in bone degradation (10). The present study therefore hypothesized that the anti-inflammatory effects of iguratimod may impact the bone destruction induced by bone metastasis. The current study detected the effects of iguratimod on bone destruction using X-rays and histological staining, and the extent of bone destruction was reduced in rats treated with iguratimod.

As aforementioned, anti-inflammatory therapy (primarily using NSAIDs) is considered to aid the relief of bone cancer pain (5). However, traditional non-selective NSAIDs are associated with gastrointestinal ulceration, renal dysfunction and impaired platelet aggregation $(8,39)$. Using COX-2 selective inhibitors may reduce the risk of gastrointestinal bleeding, but this advantage appears to reduce after 6 months of treatment, and there is also an increased risk of cardiovascular events with prolonged use of COX-2 inhibitors (39). Previous studies demonstrated that iguratimod reduced the expression levels of cytokines, potentially by suppressing NF- $\kappa \mathrm{B}$ activation without interfering with I $\mathrm{B} \alpha \alpha$ degradation (40). Previous clinical trials revealed that in rheumatoid arthritis patients who received iguratimod for 52 weeks, the adverse events were principally mild or moderate in severity, and its long-term use is safe $(13,41-43)$. According to the present study, when treated with iguratimod, not only is the mechanical allodynia of tumor-bearing rats relieved, but the bone destruction is also alleviated. As iguratimod is well-tolerated for long-term use, the current findings may provide important new insights into the treatment of bone metastasis symptoms.

In conclusion, the present study first demonstrated the effects of iguratimod on bone cancer pain and bone destruction in a rat model, but there were certain limitations. Firstly, the underlying mechanisms of iguratimod to alleviate bone cancer pain have not been extensively examined. However, future studies focus on conducting in vitro studies to further investigate these mechanisms, and the data have not yet been published. Secondly, larger-scale experiments are required to verify these effects. Finally, clinical trials are also required to test the efficacy of iguratimod in patients with bone cancer pain.

\section{Acknowledgements}

This study was supported by the National Natural Science Foundation of China (grant no. 81372852). The authors would like to thank Dr Dai Shi and Dr Xuehai Guan (Department of Anesthesiology, Tongji Hospital, Wuhan, China) for their surgical and mechanical allodynia test suggestions. The authors would also like to thank Dr Qiaochu Fu (Department of Anesthesiology, Tongji Hospital) for her provision of the Walker 256 cells. 


\section{References}

1. Roodman GD: Genes associate with abnormal bone cell activity in bone metastasis. Cancer Metastasis Rev 31: 569-578, 2012

2. Holland PM, Miller R, Jones J, Douangpanya H, Piasecki J, Roudier M and Dougall WC: Combined therapy with the RANKL inhibitor RANK-Fc and rhApo2L/TRAIL/dulanermin reduces bone lesions and skeletal tumor burden in a model of breast cancer skeletal metastasis. Cancer Biol Ther 9: 539-550, 2010

3. Zinonos I, Luo KW, Labrinidis A, Liapis V, Hay S, Panagopoulos V, Denichilo M, Ko CH, Yue GG, Lau CB, et al: Pharmacologic inhibition of bone resorption prevents cancer-induced osteolysis but enhances soft tissue metastasis in a mouse model of osteolytic breast cancer. Int J Oncol 45: 532-540, 2014.

4. Gui Q, Xu C, Zhuang L, Xia S, Chen Y, Peng P and Yu S: A new rat model of bone cancer pain produced by rat breast cancer cells implantation of the shaft of femur at the third trochanter level. Cancer Biol Ther 14: 193-199, 2013.

5. Kane CM, Hoskin P and Bennett MI: Cancer induced bone pain. BMJ 350: h315, 2015.

6. Vahtsevanos K, Kyrgidis A, Verrou E, Katodritou E, Triaridis S, Andreadis CG, Boukovinas I, Koloutsos GE, Teleioudis Z, Kitikidou K, et al: Longitudinal cohort study of risk factors in cancer patients of bisphosphonate-related osteonecrosis of the jaw. J Clin Oncol 27: 5356-5362, 2009.

7. Stopeck AT, Lipton A, Body JJ, Steger GG, Tonkin K, de Boer RH, Lichinitser M, Fujiwara Y, Yardley DA, Viniegra M, et al: Denosumab compared with zoledronic acid for the treatment of bone metastases in patients with advanced breast cancer: A randomized, double-blind study. J Clin Oncol 28: 5132-5139, 2010.

8. Koo HJ, Yoon WJ, Sohn EH, Ham YM, Jang SA, Kwon JE Jeong YJ, Kwak JH, Sohn E, Park SY, et al: The analgesic and anti-inflammatory effects of Litsea japonica fruit are mediated via suppression of NF- $\mathrm{KB}$ and JNK/p38 MAPK activation. Int Immunopharmacol 22: 84-97, 2014.

9. Yin JJ, Pollock CB and Kelly K: Mechanisms of cancer metastasis to the bone. Cell Res 15: 57-62, 2005.

10. Bussard KM, Venzon DJ and Mastro AM: Osteoblasts are a major source of inflammatory cytokines in the tumor microenvironment of bone metastatic breast cancer. J Cell Biochem 111: $1138-1148,2010$

11. Takiguchi S, Korenaga N, Inoue K, Sugi E, Kataoka Y, Matsusue K, Futagami K, Li YJ, Kukita T, Teramoto N and Iguchi $\mathrm{H}$ : Involvement of CXCL14 in osteolytic bone metastasis from lung cancer. Int J Oncol 44: 1316-1324, 2014

12. Luo Q, Sun Y, Liu W, Qian C, Jin B, Tao F, Gu Y, Wu X, Shen Y and Xu Q: A novel disease-modifying antirheumatic drug, iguratimod, ameliorates murine arthritis by blocking IL-17 signaling, distinct from methotrexate and leflunomide. J Immunol 191: 4969-4978, 2013.

13. Okamura K, Yonemoto Y, Okura C, Kobayashi Tand Takagishi K: Efficacy of the clinical use of iguratimod therapy in patients with rheumatoid arthritis. Mod Rheumatol 25: 235-240, 2015.

14. Guan XH, Fu QC, Shi D, Bu HL, Song ZP, Xiong BR, Shu B, Xiang HB, Xu B, Manyande A, et al: Activation of spinal chemokine receptor CXCR3 mediates bone cancer pain through an Akt-ERK crosstalk pathway in rats. Exp Neurol 263: 39-49, 2015.

15. Mao-Ying QL, Zhao J, Dong ZQ, Wang J, Yu J, Yan MF, Zhang YQ, Wu GC and Wang YQ: A rat model of bone cancer pain induced by intra-tibia inoculation of Walker 256 mammary gland carcinoma cells. Biochem Biophys Res Commun 345: 1292-1298, 2006.

16. Du F, Lü LJ, Fu Q, Dai M, Teng JL, Fan W, Chen SL, Ye P, Shen N, Huang XF, et al: T-614, a novel immunomodulator, attenuates joint inflammation and articular damage in collagen-induced arthritis. Arthritis Res Ther 10: R136, 2008.

17. Xu B, Guan XH, Yu JX, Lv J, Zhang HX, Fu QC, Xiang HB Bu HL, Shi D, Shu B, et al: Activation of spinal phosphatidylinositol 3-kinase/protein kinase B mediates pain behavior induced by plantar incision in mice. Exp Neurol 255: 71-82, 2014.

18. Pogatzki EM and Raja SN: A mouse model of incisional pain. Anesthesiology 99: 1023-1027, 2003.

19. Bloom AP,Jimenez-Andrade JM,Taylor RN, Castañeda-Corral G, Kaczmarska MJ, Freeman KT, Coughlin KA, Ghilardi JR, Kuskowski MA and Mantyh PW: Breast cancer-induced bone remodeling, skeletal pain, and sprouting of sensory nerve fibers. J Pain 12: 698-711, 2011.

20. Medhurst SJ, Walker K, Bowes M, Kidd BL, Glatt M, Muller M, Hattenberger M, Vaxelaire J, O'Reilly T, Wotherspoon G, et al: A rat model of bone cancer pain. Pain 96: 129-140, 2002.
21. Gao YJ and Ji RR: c-Fos and pERK, which is a better marker for neuronal activation and central sensitization after noxious stimulation and tissue injury? Open Pain J 2: 11-17, 2009.

22. Wang LN, Yao M, Yang JP, Peng J, Peng Y, Li CF, Zhang YB, Ji FH, Cheng H, Xu QN, et al: Cancer-induced bone pain sequentially activates the ERK/MAPK pathway in different cell types in the rat spinal cord. Mol Pain 7: 48, 2011.

23. Wang XW, Li TT, Zhao J, Mao-Ying QL, Zhang H, Hu S, Li Q, Mi WL, Wu GC, Zhang YQ and Wang YQ: Extracellular signal-regulated kinase activation in spinal astrocytes and microglia contributes to cancer-induced bone pain in rats. Neuroscience 217: 172-181, 2012.

24. Doré-Savard L, Otis V, Belleville K, Lemire M, Archambault M, Tremblay L, Beaudoin JF, Beaudet N, Lecomte R, Lepage M, et al: Behavioral, medical imaging and histopathological features of a new rat model of bone cancer pain. PLoS One 5: e13774, 2010.

25. Kingsley LA, Fournier PG, Chirgwin JM and Guise TA: Molecular biology of bone metastasis. Mol Cancer Ther 6 : 2609-2617, 2007

26. Sterling JA, Edwards JR, Martin TJ and Mundy GR: Advances in the biology of bone metastasis: How the skeleton affects tumor behavior. Bone 48: 6-15, 2011.

27. Siclari VA, Guise TA and Chirgwin JM: Molecular interactions between breast cancer cells and the bone microenvironment drive skeletal metastases. Cancer Metastasis Rev 25: 621-633, 2006.

28. Mantyh P: Bone cancer pain: Causes, consequences, and therapeutic opportunities. Pain 154 (Suppl 1): S54-S62, 2013.

29. Sosnoski DM, Krishnan V, Kraemer WJ, Dunn-Lewis C and Mastro AM: Changes in cytokines of the bone microenvironment during breast cancer metastasis. Int J Breast Cancer 2012: $160265,2012$.

30. Jimenez-Andrade JM, Mantyh WG, Bloom AP, Ferng AS, Geffre CP and Mantyh PW: Bone cancer pain. Ann N Y Acad Sci 1198: 173-181, 2010

31. Yoneda T, Hata K, Nakanishi M, Nagae M, Nagayama T, Wakabayashi H, Nishisho T, Sakurai $T$ and Hiraga $T$ : Involvement of acidic microenvironment in the pathophysiology of cancer-associated bone pain. Bone 48: 100-105, 2011.

32. Clark AK, Old EA and Malcangio M: Neuropathic pain and cytokines: Current perspectives. J Pain Res 6: 803-814, 2013.

33. Ellis A and Bennett DL: Neuroinflammation and the generation of neuropathic pain. Br J Anaesth 111: 26-37, 2013.

34. Falk S and Dickenson AH: Pain and nociception: Mechanisms of cancer-induced bone pain. J Clin Oncol 32: 1647-1654, 2014.

35. Du F, Lü LJ, Teng JL, Shen N, Ye P and Bao CD: T-614 alters the production of matrix metalloproteinases (MMP-1 andMMP-3) and inhibits the migratory expansion of rheumatoid synovial fibroblasts, in vitro. Int Immunopharmacol 13: 54-60, 2012.

36. David Roodman G: Role of stromal-derived cytokines and growth factors in bone metastasis. Cancer 97 (3 Suppl): S733-S738, 2003

37. Ara T and Declerck YA: Interleukin-6 in bone metastasis and cancer progression. Eur J Cancer 46: 1223-1231, 2010.

38. Ara T, Song L, Shimada H, Keshelava N, Russell HV, Metelitsa LS, Groshen SG, Seeger RC and DeClerck YA: Interleukin-6 in the bone marrow microenvironment promotes the growth and survival of neuroblastoma cells. Cancer Res 69: 329-337, 2009.

39. Paice JA and Ferrell B: The management of cancer pain. CA Cancer J Clin 61: 157-182, 2011.

40. Aikawa Y, Yamamoto $M$, Yamamoto $T$, Morimoto $\mathrm{K}$ and Tanaka K: An anti-rheumatic agent T-614 inhibits NF-kappaB activation in LPS- and TNF-alpha-stimulated THP-1 cells without interfering with IkappaBalpha degradation. Inflamm Res 51: 188-194, 2002

41. Okamura K, Yonemoto Y, Suto T, Okura C and Takagishi K: Efficacy at 52 weeks of daily clinical use of iguratimod in patients with rheumatoid arthritis. Mod Rheumatol 25: 534-539, 2015.

42. Hara M, Abe T, Sugawara S, Mizushima Y, Hoshi K, Irimajiri S, Hashimoto H, Yoshino S, Matsui N and Nobunaga M: Long-term safety study of iguratimod in patients with rheumatoid arthritis. Mod Rheumatol 17: 10-16, 2007.

43. Hara M, Ishiguro N, Katayama K, Kondo M, Sumida T, Mimori T, Soen S, Nagai K, Yamaguchi T and Yamamoto K; Iguratimod-Clinical Study Group: Safety and efficacy of combination therapy of iguratimod with methotrexate for patients with active rheumatoid arthritis with an inadequate response to methotrexate: An open-label extension of a randomized, double-blind, placebo-controlled trial. Mod Rheumatol 24: 410-418, 2014. 